\title{
Is stereotactic large-core needle biopsy beneficial prior to surgical treatment in BI- RADS 5 lesions?
}

\author{
H.M. Verkooijen ${ }^{1}$, G. Vlastos ${ }^{2}$, and K. Kinkel-Trugli ${ }^{3}$ \\ ${ }^{1}$ Geneva Cancer Registry, Institute for Social and Preventive Medicine, Geneva University; ${ }^{2}$ Department of Gyne- \\ cology and Obstetrics, Geneva University Hospitals; ${ }^{3}$ Department of Radiology, Cliniques des Grangettes, Chêne- \\ Bougeries, Switzerland
}

With great interest we have read the article of Hoorntje et al. [1] addressing the usefulness of stereotactic largecore needle biopsy prior to surgery in BI-RADS 5 lesions. We fully agree with the authors that the far majority of women with BI-RADS 5 lesions presenting as non-calcified mass densities will not need stereotactic large core needle biopsy. However, we do not agree with the authors' suggestions of immediate surgery with sentinel node biopsy in case of BI-RADS 5 lesions presenting as a density without calcifications.

International guidelines state that at least $90 \%$ of women with breast cancer should have a pre-operative diagnosis of malignancy (fine needle biopsy or core biopsy reported as definitely malignant) [2]. Pre-operative histological diagnosis allows planning of definite treatment, i.e. surgery aiming for negative margins, sentinel lymph node biopsy or axillary lymph node dissection and in some cases neoadjuvant chemotherapy. In addition, pre-operative histology may diminish the patient's uncertainty and gives the treating physician the possibility to provide the necessary information concerning the disease and its treatment. We think that today, every woman with an abnormal mammography should receive a definitive diagnosis without delay, in any case before admission to surgery (which might take weeks, particularly in countries with long waiting lists).

Does that mean that all women with BI-RADS 5 lesions presenting as mass densities should undergo stereotactic large core needle biopsy? Probably anyone will agree that the answer is no. The far majority of mammographic densities can be targeted by the more convenient and less expensive ultrasound guided largecore needle biopsy.

In addition, we find the calculation at the end of the results section somewhat difficult to follow. The authors state that 'scenario II' (immediate surgery with sentinel lymph node biopsy for BI-RADS 5 lesions presenting as mass densities) could prevent $65 \%$ (i.e. 154 out of 238 ) of the stereotactic large core needle biopsies. We believe that this figure is largely overestimated. In the discussion section, the authors say that in case of planned mastectomy, pre-operative stereotactic large-core needle biopsy is indicated. In this study, $32 \%$ of the patients had mastectomy as primary surgical intervention. Subsequently, one third $( \pm 51)$ of the 154 'preventable' stereotactic large core needle biopsies would not be so 'preventable' after all. A preventable proportion of $43 \%$ (103 out of 238) stereotactic large-core needle biopsies would be more realistic. Multifocality or occurrence of lesions otherwise not suitable for sentinel lymph node biopsy could even lower this number.

One might conclude that the prevention of some 100 preoperative biopsies (ultrasound or stereotactic guided) makes up for four unnecessarily over-treated benign lesions. However, when taking into account the delay in providing an anxious patient with a definite diagnosis and information concerning her disease, we're not so sure if the balance still favours 'scenario II'.

\section{References}

1. Hoorntje LE, Peeters PHM, Mali WPThM, Borel Rinkes IHM. Is stereotactic large-core needle biopsy beneficial prior to surgical treatment in BI-RADS 5 lesions. Breast Cancer Res Treatm 86: 165-170, 2004

2. http://www.eusoma.org/Index.aspx

Address for offprints and correspondence: H. M. Verkooijen, Institute for Social and Preventive Medicine, Geneva Cancer Registry, 55 Boulevard de la Cluse, Geneva 1205, Switzerland E-mail: Lenny.Verkoojen@imsp.unige.ch 